\title{
Erratum: Robust entanglement with three-dimensional nonreciprocal photonic topological
} insulators [Phys. Rev. A 95, 063807 (2017)]

S. Ali Hassani Gangaraj, George W. Hanson, and Mauro Antezza

Q (Received 23 May 2018; published 11 June 2018)

DOI: 10.1103/PhysRevA.97.069902

There were two typographical errors in Eq. (A1) in this paper. The correct equation is

$$
2 i \frac{\omega^{2}}{c^{2}} \int d^{3} r^{\prime \prime} \mathbf{G}\left(\mathbf{r}, \mathbf{r}^{\prime \prime}, \omega\right) \cdot \mathbf{T}\left(\mathbf{r}^{\prime \prime}, \omega\right) \cdot \mathbf{T}^{\dagger}\left(\mathbf{r}^{\prime \prime}, \omega\right) \mathbf{G}^{\dagger}\left(\mathbf{r}^{\prime}, \mathbf{r}^{\prime \prime}, \omega\right)=\mathbf{G}\left(\mathbf{r}, \mathbf{r}^{\prime}, \omega\right)-\mathbf{G}^{\dagger}\left(\mathbf{r}^{\prime}, \mathbf{r}, \omega\right) .
$$

